\title{
The Role of Ultra-widefield Retinal Imaging as a Standard Assessment Tool in the Cataract Practice
}

\author{
Kerry K Assil ${ }^{1}$ and V Nicholas Batra²
}

1. Assil Eye Institute, Beverly Hills, California, US; 2. Batra Vision, San Leandro, California, US

C omprehensive evaluation of retinal health prior to and following cataract surgery is critical to supporting optimal outcomes. In addition to the importance of identifying retinal pathology that may prevent or delay cataract surgery, continuous advances in refractive intraocular lens technology and cataract surgical technique, coupled with increasingly high expectations regarding visual outcomes among younger patients, make the consideration of long-term quality of vision paramount in the cataract assessment. Optos ${ }^{\circledR}$ ultrawidefield retinal imaging supports this clinical objective by providing imaging standardization in a streamlined, patient-friendly exam process, supporting robust documentation that facilitates mapping of disease progression, and offering potential economic advantages in a resourceconstrained environment.

\section{Keywords}

Ultra-widefield retinal imaging, UWF, cataract surgery, multifocal IOL, retinal periphery

Disclosure: Kerry K Assil has nothing to disclose in relation to this article. $V$ Nicholas Batra participated in a panel case presentation sponsored by Optos

Acknowledgments: Editorial assistance was provided by BioComm Network, Inc.

Compliance with Ethics: This study a review of the literature and did not involve any studies with human or animal subjects performed by any of the authors.

Authorship: All named authors meet the International Committee of Medical Journal Editors (ICMJE) criteria for authorship of this manuscript, take responsibility for the integrity of the work as a whole, and have given final approval to the version to be published.

open Access: This article is published under the Creative Commons Attribution Noncommercial License, which permits any noncommercial use, distribution, adaptation, and reproduction provided the original author(s) and source are given appropriate credit.

Received: February 6, 2017

Accepted: March 22, 2017

Citation: US Ophthalmic Review, 2017;10(1):31-4

Corresponding Author: Kerry K Assil,

450 N. Roxbury Dr., 3rd Floor, Beverly Hills,

CA 90210, US. E: kerry@assileye.com

Support: The publication of this article was supported by Optos. The views and opinions expressed are those of the authors and do not necessarily reflect those of Optos.
Due in part to continuous improvement in the safety, speed, and refractive outcomes of cataract surgery, the number of cataract procedures has increased steadily over the past 30 years, and the percentage of patients undergoing the procedure at a younger age is also increasing. These trends were documented in a frequently cited population-based study of Omsted County, Minnesota, that compared rates of cataract surgery between 2005 and 2011 to those in the prior 25 years. Data on more than 8,000 cataract procedures in the most recent period revealed that the incidence of cataract surgery had increased significantly, the interval between first and second eye surgery narrowed significantly, and $19 \%$ of patients elected cataract surgery before age $65 .{ }^{1}$ This rise in cataract surgery rates will continue to accelerate as the US population ages; the number of Americans over age 65 is expected to nearly double by 2050 , to 83.7 million. $^{2}$

Along with the increasing volume driven by this large demographic wave, there are a number of important implications of the shift to earlier cataract surgery and the increasing use of sophisticated premium intraocular lens (IOL) technologies. Perhaps the most significant of these is that many patients will live for decades after the procedure. These patients have high expectations of their immediate postoperative visual outcomes and also expect to continue to see well as they age. With sustained quality of vision the main therapeutic objective, it has become paramount to perform thorough retinal exams pre-surgically and postoperatively to identify pre-existing retinal disease that might prevent, delay, or alter surgery, to anticipate future retinal problems-particularly to the degree that this prospect may affect lens choice-and to provide an initial, post-cataract baseline for monitoring future pathological changes. In this review, we will describe the integration into our pre- and post-cataract surgery assessments of a specific imaging technology that has shown utility in retinal disease detection and management for both the general ophthalmologist and the retinal specialist. In our experience, ultra-widefield (UWF'M) retinal imaging (Optos plc., Dunfermline, Scotland) and the clinical and practical value of the high-resolution, digital optomap ${ }^{\circledR}$ images they produce, have complemented the information available to us from standard approaches, including conventional fundus examination and optical coherence tomography (OCT), thereby improving our ability to rapidly and accurately identify retinal pathology, facilitating consultation with retina colleagues, and resulting in enhanced patient care, increased patient satisfaction, and greater efficiency within our practices. As a result, Optos ${ }^{\circledR}$ UWF retinal imaging has become a routine component of perioperative evaluation in our cataract cases, based on the unique advantages of the technology, and we believe that other cataract practices may benefit from considering this approach. 
Figure 1: optomap of proliferative diabetic retinopathy visible though a cataract

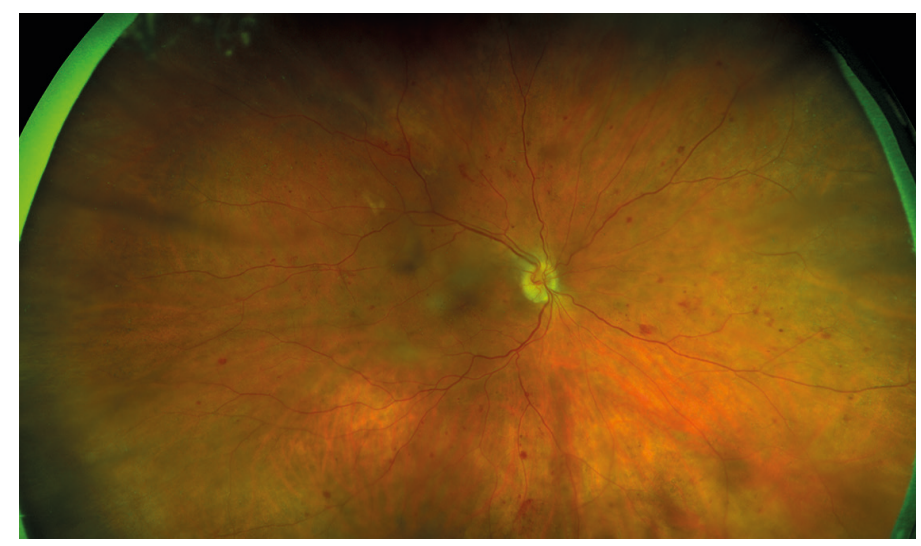

\section{Relevant capabilities of ultra-widefield retinal imaging for the cataract practice}

As occurred with the introduction of OCT, now an essential tool in many cataract practices, UWF retinal imaging is providing new capabilities that are helping to evolve the standard of care. Several platforms for examining the retinal periphery have been developed, including the portable, contact-based RetCam (Natus Medical, Plesanton, California, US) and contact (Staurenghi retinal lens-Ocular Instruments, Washington, US) and non-contact lens additions to the Heidelberg Spectralis (Heidelberg Engineering, Heidelberg, Germany), but the Optos systems offer the maximum field of view of any available retinal imaging device. ${ }^{3}$ Optos UWF devices are also easier to use and capture images more quickly than other platforms. A single, non-contact capture can show 200-degrees or $82 \%$ of the retina, often extending beyond the vortex veins and equator. A number of studies have confirmed that Optos UWF imaging helps clinicians identify pathology associated with a wide array of retinal diseases, including non-infectious uveitis and vasculitis, that can be missed with conventional imaging techniques. ${ }^{4-6} \mathrm{~A}$ review of data from more than 3 years of research with UWF imaging confirmed that that $66 \%$ of clinically relevant retinal pathology is found outside the reach of conventional fundus photography.

In our perioperative assessments, the functional capabilities of Optos systems make UWF imaging a useful adjunct to OCT and other standard examination tools, particularly in providing an efficient way to examine the retinal periphery. Each system is built around two low-power lasers and purpose-built optical technology that permit high-resolution image capture through a pupil as small as $2 \mathrm{~mm}$. Since mydriasis is not necessary for excellent visualization, imaging is possible in eyes that cannot be dilated, such as those with anatomic narrow angles, pseudoexfoliation, iris trauma, or uveitis. Considering that many cataract patients are somewhat more light sensitive during the preoperative time period and given the relatively high frequency with which dilated fundus evaluations occur in such patients (for further assessment of dysphotopsias, floaters, visual blur or just routine surveillance), the potential benefits of UWF imaging, including screening sensitivity and efficiency, may be further amplified in this particular group. From the clinician's perspective, conventional retinal imaging can be challenging in patients with cataracts due to light scatter off the cataract. However, the Optos system scanning laser ophthalmoscopy (SLO) technology
Figure 2: Screen shots demonstrating the versatility of optomap in the pre-surgical examination; the color images identified retinitis pigmentosa

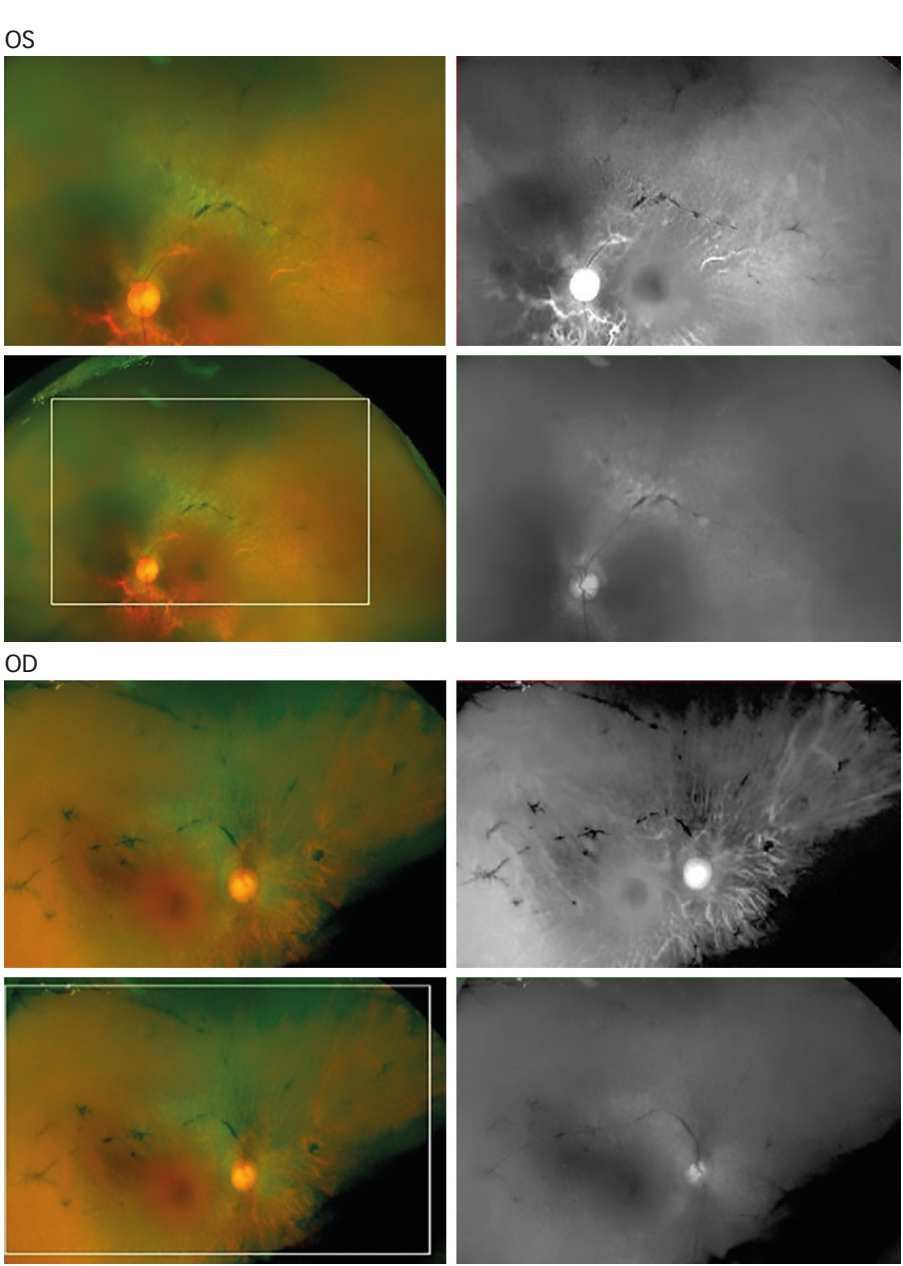

Each set of four images (OS/OD) is from a single capture and shows (clockwise from bottom left): 1) original UWF color image; 2) zoomed view of the pathology, note the bone spicule pattern; 3) IR/choroidal view achieved by setting the blend feature to $100 \%$ red; 4) red-free/surface view achieved by setting the blend feature to $100 \%$ green.

can effectively penetrate most media opacities, allowing adequate visualization through cataracts, vitreous hemorrhages, corneal scars, and gas bubbles or silicone oil (see Figure 1).

The sensitivity and clinical value of UWF retinal imaging technology in the identification and assessment of diabetic retinopathy is well documented. ${ }^{8-11}$ It has also been evaluated extensively in a spectrum of other retinal diseases, particularly retinal vasculopathies..$^{12,13}$ These studies have contributed to the growing significance of peripheral findings to disease management. The ease and speed of optomap image capture makes it feasible to incorporate them into routine exams with the result that overall exam time is reduced and patient satisfaction increased.

Beyond rapid image capture, UWF retinal imaging provides additional efficiency advantages for the cataract practice. The OptosAdvance ${ }^{\mathrm{TM}}$ image management software permits cross-registration of multimodal images, the manipulation and annotation of images, and the importation of Digital Imaging and Communications in Medicine (DICOM) compatible images from other diagnostic equipment, all of which can be viewed on 
Figure 3: optomap demonstrating age-related macular degeneration imaged through a cataract allowing visibility of the central atrophy and pigmentary changes in the periphery

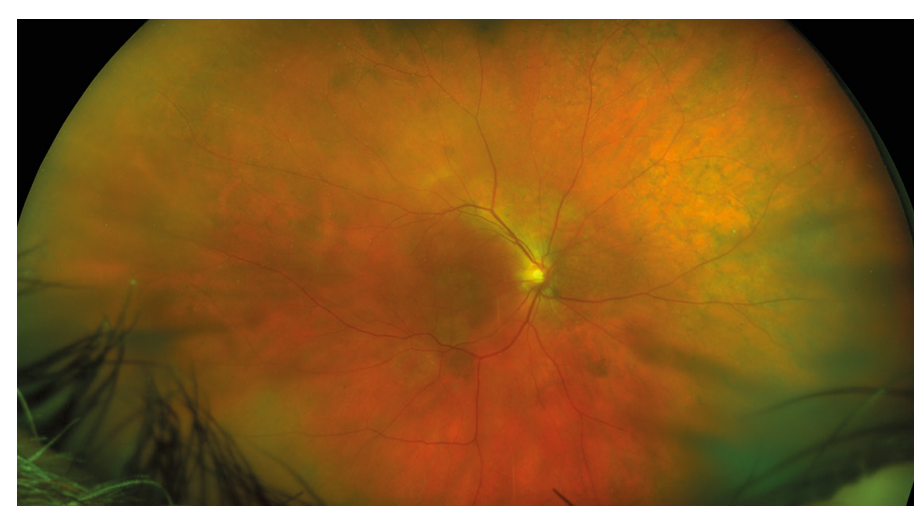

any networked computer screen or tablet. Image storage and sharing between offices or practices is managed through a cloud-based archive, minimizing storage challenges for the individual office. Remote offices are able to review the optomap files over the internet, without the requirement of Optos hardware or software. OptosAdvance also provides a $3 \mathrm{D}$ projection of the globe and major anatomical structures, which is very useful in educating patients about the health of their eyes and discussing IOL options.

\section{The utility of ultra-widefield retinal imaging in the cataract assessment and follow-up Pre-existing retinal pathology}

Although there are few outright contraindications for cataract surgery, other than a low endothelial cell count, the detection and/or careful evaluation of pre-existing retinal pathology is critical to identify patients who may need treatment of retinal disease prior to cataract surgery or may require particular care during the procedure, both to prevent worsening of the underlying retinal condition and to optimize the outcomes of the cataract procedure. UWF is very useful in identifying and documenting pathologies such as lattice degeneration, macular holes or tears, and cobblestone degeneration, all of which carry a higher risk of retinal detachment.

Cataract evaluation often uncovers the first indications of retinal pathology in a patient, with preoperative referral providing confirmation of a diagnosis. In this case (see Figure 2), for example, multimodal UWF imaging revealed retinitis pigmentosa (RP) in a 62-year-old patient referred for blurry vision and cataract. The patient was subsequently seen by a retina specialist and cleared for cataract surgery. Because of the RP diagnosis, we chose a monofocal implant. Following cataract extraction, the patient is $20 / 30$ uncorrected OU and is very happy with his vision. The optomap images were also useful in the process of educating the patient about RP.

In most cases, pre-existing retinal disease, including age-related macular degeneration (AMD) and diabetic retinopathy, does not prevent the patient from undergoing cataract surgery. Large studies have shown that cataract surgery in these patients can provide sustained improvements in visual acuity. . 1,15 However, the impact of the procedure on the course of retinal disease remains controversial. Some evidence suggests increases in both the incidence and progression rate of diabetic retinopathy at 1 year after phacoemulsification cataract surgery,
Figure 4: optomap af image of the same patient illustrates two levels of damage

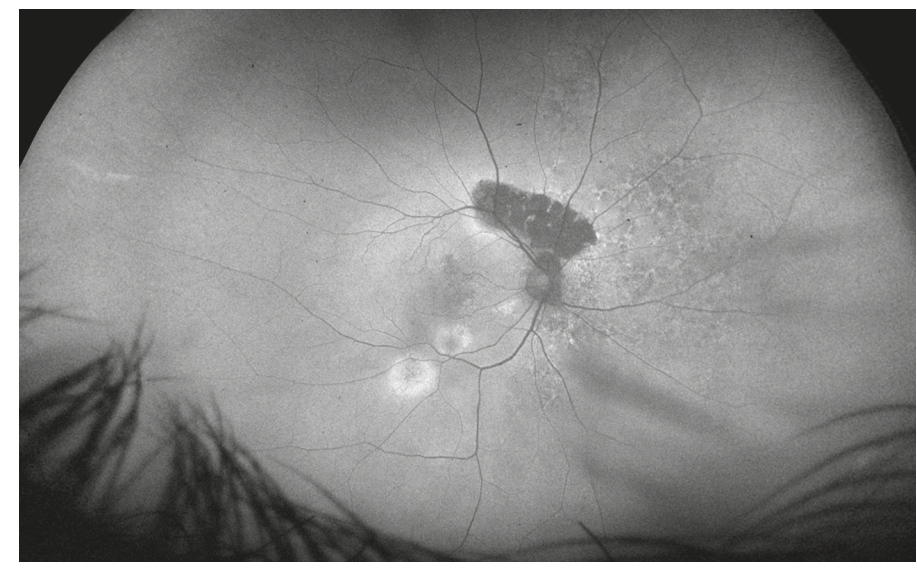

Hypoautofluorescence, a decreased signal, indicates a complete loss of function. Hyperautofluorescence, an increased signal, shows areas of dysfunction, but not loss. The widespread extent of retinal pigmented epithelial damage can be tracked over time.

and findings are inconsistent from large studies about the association between cataract surgery and long-term risk for the development of either early or late AMD. ${ }^{16-18}$ It is clearer that the risk of post-surgical cystoid macular edema is increased in patients with a history of retinal vein occlusion or epiretinal membrane. ${ }^{19}$

Given the totality of the available evidence and our own experience, we are very conservative in the presence of retinal findings and maintain a low threshold for referral to a retinal specialist for further evaluation, including a dilated fundus exam and OCT, and potential treatment (see Figures 3 and 4). In these situations, the ability to rapidly and easily share an optomap image using the cloud facilitates inter-office communication and efficient patient management. In most of these cases, we are ultimately able to proceed with cataract surgery but may take additional measures in the postoperative phase to monitor the patient and aggressively control inflammation.

\section{Lens choice considerations}

Pathology in the macula evident on UWF is also important to the consideration of premium lens options, particularly multi-focal IOLs. Because of their design, these lenses can be associated with a decrease in contrast sensitivity and photic phenomena such as glare and haloseffects that can be compounded by retinal disease..$^{20,21}$ They may also result in impaired visualization of the retina in subsequent retinal surgery. If evidence of diabetic retinopathy, AMD, or epiretinal membranes is identified on UWF, additional assessment, including macular function, should be conducted in consultation with the retina specialist to help determine the expected course of the disease and the long-term suitability of the lens choice. As lens technology continues to improve, providing greater degrees of spectacle independence with fewer trade-offs in vision quality, and data on long-term performance multi-focal IOLs in the presence of retinal disease is collected, these concerns may eventually be obviated.

\section{Postoperative follow-up}

While large, prospective studies of the use of UWF in post-cataract monitoring have not yet been performed, Faberowski et al. have demonstrated that non-mydriatic color UWF imaging can be used 
effectively to screen for retinal pathology in asymptomatic patients following cataract extraction. ${ }^{22}$ In their retrospective, observational case series in 76 eyes of 58 consecutive patients, UWF imaging provided good visualization of the peripheral retina in more than $90 \%$ of the patients and did not reveal any new retinal tears or detachments, including in patients with prior history of retinal pathology. Another group has demonstrated that non-mydriatic UWF imaging of patients after cataract surgery provides a rate of detection for retinal lesions comparable to mydriatic slit lamp and mydriatic Goldmann three-mirror contact lens examinations, while offering more convenience for the practice and comfort for the patient. ${ }^{23}$

We typically repeat optomap imaging at 2 weeks following surgery, even in asymptomatic patients. This gives us the ability to screen for any fundus changes while obtaining the first baseline UWF image of the retina with the cataractous lens removed.

\section{Advantages of ultra-widefield retinal imaging in the cataract practice}

In our experience, the benefits of integrating UWF retinal imaging into cataract assessment and follow-up can be grouped into five general areas:

\section{Standardization}

The use of the standardized UWF optomap image, within and between practices, helps reduce inter-observer variability and facilitates diagnostic and management consensus. The ability to overlay different imaging modalities or compare prior images is very useful in detecting and monitoring retinal pathology.

\section{Streamlining retinal assessment}

The ability to rapidly capture a high-resolution digital image in a patientfriendly manner makes UWF retinal imaging an excellent evaluation tool, obviating the need, time, and cost of a dilated fundus exam unless findings are made. optomap images can be easily captured by a tech and are immediately available to the surgeon for evaluation.

\section{Robust documentation}

The standardized, digital optomap image is easily stored, annotated, and compared, providing a clinically useful component of the patient's record. The use of UWF enhances documentation of retinal pathology, cataract opacity, implant choice, and postoperative results

\section{Mapping progression}

Evaluation and post-op UWF images create an important baseline for ongoing monitoring of disease progression in patients with evident retinal pathology. The ability to compare a standardized image over time, annotate images, and toggle between imaging modalities provides additional assurance that pathological changes can be identified.

\section{Economic advantages}

The efficiency and ease of use of UWF imaging potentially allows the evaluation of a larger population of patients over a shorter period of time. For us, this has translated into improved patient flow and surgical volume. In addition, in some cases, documentation of peripheral pathology outside the reach of conventional cameras represents a reimbursable service that improves the economic equation for the practice. Offering UWF retinal imaging on a modest, fee-for-service basis may have similar benefits while ensuring that most patients enjoy the clinical benefits of a baseline optomap.

\section{Conclusion}

The increasing number of cataract patients-particularly those under 65-and heightened patient expectations about postoperative visual outcomes are placing new demands on the cataract practice's clinical rigor and resource allocation. The integration of Optos UWF retinal imaging into our preoperative assessment and postoperative follow-up protocols is helping to address these issues by offering an efficient, economical, and patient-friendly evaluation tool for retinal pathology, providing a consistent point of reference between clinicians, and helping to document retinal changes over time to help protect the patient's long-term quality of vision. Additional experience by cataract surgeons and evidence from formal studies will help further define the potential contribution of UWF retinal imaging to enhancing immediate and long-term surgical outcomes.
1. Gollogly HE, Hodge DO, St Sauver JL, Erie JC, Increasing incidence of cataract surgery: population-based study, I Cataract Refract Surg, 2013;39:1383-9.

2. Ortman JM, Velkoff VA, Hogan $\mathrm{H}$, An aging nation: the older population in the United States. Current population reports, May 2014, U.S. Census Bureau.

3. Witmer MT, Parlitsis G, Patel S, et al., Comparison of ultra-widefield fluorescein angiography with the Heidelberg Spectralis ${ }^{\varpi}$ noncontact ultra-widefield module versus the Optos ${ }^{\oplus}$ Optomap $^{\oplus}$ Clin Ophthalmol, 2013;7:389-94.

4. Leder HA, Campbell JP, Sepah YJ, et al., Ultra-wide-field retinal imaging in the management of non-infectious retinal vasculitis, J Ophth Inflamm Infect, 2013:3:30

5. Nicholson BP Nigam D, Miller D, et al. Comparison of wide-field fluorescein angiography and nine-field montage angiography in fluorescein angiography and nine-field montas

6. Hong BK, Khanamiri HN, Rao NA, Role of ultra-widefield fluorescein angiography in the management of uveitis, Can J Ophthalmol, 2013;48:489-93.

7. Optos. Data on file.

8. Kernt M, Hadi I, Pinter F, et al., Assessment of diabetic retinopathy using nonmydriatic ultra-widefield scanning laser ophthalmoscopy (optomap) compared with ETDRS 7-field stereo ophthalmoscopy (optomap) compared with E

9. Silva PS, Cavallerano JD, Sun JK, et al., Nonmydriatic ultrawide field retinal imaging compared with dilated standard 7-field $35-\mathrm{mm}$ photography and retinal specialist examination for evaluation of diabetic retinopathy, Am J Ophthalmol, 2012;154:549-59.

10. Liegl R, Lieg| K, Ceklic L, et al., Nonmydriatic ultra-wide-field scanning laser ophthalmoscopy (optomap) versus two-field fundus photography in diabetic retinopathy, Ophthalmologica, 2014:231:31-6.

11. Wessel MM, Aaker GD, Parlitsis G, et al., Ultra-wide-field angiography improves the detection and classification of diabetic retinopathy, Retina, 2012;32:785-91.

12. Tsui I, Franco-Cardenas V, Hubschman J, et al., Ultra wide field fluorescein angiography can detect macular pathology in central retinal vein occlusion, Ophthalmic Surg Lasers Imaging 2012;43:257-62.

13. Singer $M$, Tan $C S$, Bell $D$, et al., Area of peripheral retina nonperfusion and treatment response in branch and central nonperfusion and treatment response in branc
retinal vein occlusion, Retina, 2014:34:1736-42.

14. Forooghian F, Agron E, Clemons TE, et al., Visual acuity outcomes after cataract surgery in patients with age-related macular degeneration: age-related eye disease study report no. 27, Ophthalmology, 2009;116:2093-100.

15. Chew EY, Benson WE, Remaley NA, et al., Results after lens extraction in patients with diabetic retinopathy: early treatment diabetic retinopathy study report number 25, Arch Ophthalmol 1999:117:1600-6.

16. Hong T, Mitchell P, de Loryn T, et al., Development and progression of diabetic retinopathy 12 months afte phacoemulsification cataract surgery, Ophthalmology, 2009;116:1510-14

17. Klein BEK, Howard KP, Lee KE, et al., The relationship of cataract and cataract extraction to age-related macular degeneration: the Beaver Dam eye study, Ophthalmology, 2012;119:1628-33.

18. Cugati $S$, Mitchell $P$, Rochtchina $E$, et al., Cataract surgery and the 10 -year incidence of age-related maculopathy: the Blue Mountains eye study, Ophthalmology, 2006;113:2020-25.

19. Henderson BA, Kim JY, Ament CS, et al., Clinical pseudophakic cystoid macular edema. Risk factors for development and duration after treatment, $I$ Cataract Refract Surg 2007:33:1550-58.

20. Braga-Mele R, Chang D, Dewey S, et al., Multifocal intraocular lenses: Relative indications and contraindications for

21. De Vries NE, Nuijts RMMA, Multifocal intraocular lenses in cataract surgery: literature review of benefits and sides effects, J Cataract Refract Surg, 2013;39:268-78

22. Faberowski N, Quiroz-Mercado H, Gonzalez C, et al., Retina evaluation with non mydriatic ultra wide field color imaging after cataract surgeries in asymptomatic patients, Invest Ophthalmo Vis Sci, 2013:54:2997.

23. Peng J, Zhang $\mathrm{Q}$, Jin $\mathrm{H}-\mathrm{Y}$, et al., Ultra-wide field imaging system and traditional retinal examinations for screening fundus changes after cataract surgery, Int J Ophthalmol 2016:9:1299-303. 